\title{
Abundância, distribuição espacial de ninhos de abelhas Meliponina (Hymenoptera, Apidae, Apini) e espécies vegetais utilizadas para nidificação em áreas de cerrado do Maranhão
}

\author{
Bruna D. V. Serra ${ }^{1}$, Murilo S. Drummond ${ }^{2}$, Lenira de M. Lacerda² \& Ivan P. Akatsu ${ }^{3}$
}

1. Departamento de Biologia Geral, Universidade Federal de Viçosa, Av. P. H. Rholfs, s/n, 36570-000 Viçosa, MG, Brasil. (bruna@amavida.org.br)

2. Departamento de Biologia, Universidade Federal do Maranhão, Av. dos Portugueses, s/n, Bacanga, 65080-040 São Luís, MA, Brasil. (msdrumm@uol.com.br; leniralacerda@uol.com.br)

3. Departamento de Biologia, Faculdade de Filosofia Ciências e Letras de Ribeirão Preto, USP, Av. Bandeirantes, 3900, 14040-901 Ribeirão Preto, SP, Brasil. (akatsu@usp.br)

\begin{abstract}
Abundance, spatial distribution of Meliponina bees' nests (Hymenoptera, Apidae, Apini) and plant species used as nesting sites in savanna areas of Maranhão, Brazil. This study aimed at identifying the stingless bees species (Hymenoptera: Apidae, Apini, Meliponina) those were found in three study sites of cerrado, northeastern Brazil. The study also aimed identifying the plant species those were used as nesting sites by the stingless bees and verifying their characteristics. We intended to verify abundance and spatial distribution of the nests and patterns of the plants used as nesting sites. Seventy-three nests of 15 species were found. The most abundant species were Partamona chapadicola Pedro \& Camargo, 2003 (34,25\%) and Oxytrigona sp. 2 (20,55\%). Eleven tree species were used as nesting sites. The most common host tree species was Qualea parviflora (Vochysiaceae) where 38,36\% of the nests ( $\mathrm{n}=28$ ) nestled, followed by Salvertia convallariodora (Vochysiaceae) $(\mathrm{n}=17,23,29 \%)$. The $95 \%$ confidence interval of DHB was between $36,21 \mathrm{~cm}$ and $41,68 \mathrm{~cm}$. This interval is related with older trees that should have more hollows to be used as nesting sites. This process might be occurring with $S$. convallariodora and $Q$. parviflora. The patterns of spatial distribution for study sites 1 and 2 were random and the pattern for study site 3 was uniform. Random pattern would be a sign for absence of competition. On the other hand uniform pattern would indicate competition.
\end{abstract}

KEYWORDS. Meliponina, fragmentation, Cerrado, nesting sites, spatial distribution.

RESUMO. O trabalho objetivou identificar as espécies de abelhas sem ferrão (Hymenoptera, Apidae, Apini, Meliponina) presentes em três áreas de cerrado no Maranhão, nordeste do Brasil, por meio do levantamento de seus ninhos. Também foi objetivo do trabalho identificar e caracterizar os substratos vegetais utilizados como locais de nidificação. Pretendeu-se averiguar a abundância e a distribuição espacial de ninhos, bem como padrões de uso dos substratos para nidificação. Foram encontrados 73 ninhos pertencentes a 15 espécies. As espécies mais abundantes foram Partamona chapadicola Pedro \& Camargo, 2003 (34,25\%) e Oxytrigona sp. 2 (20,55\%). Identificaramse 11 espécies vegetais utilizadas para construção dos ninhos. O substrato de nidificação mais freqüente foi Qualea parviflora (Vochysiaceae), na qual encontrou-se $38,36 \%$ do total de ninhos $(n=28)$, seguido por Salvertia convallariodora (Vochysiaceae) $(n=17 ; 23,29 \%)$. O intervalo de confiança de $95 \%$ para o DAP situou-se entre $36,21 \mathrm{~cm}$ a $41,68 \mathrm{~cm}$. Este intervalo representaria árvores mais velhas que teriam mais cavidades disponíveis para nidificação, o que poderia ser o caso de $S$. convallariodora e Q. parviflora. O padrão de dispersão dos substratos com ninhos mostrou-se aleatório nas áreas 1 e 2 e uniforme na área 3. Padrões de distribuição aleatórios seriam um indício da ausência de competição e padrões uniformes indicariam competição.

PALAVRAS-CHAVE. Meliponina, fragmentação, cerrado, substratos de nidificação, distribuição espacial.

Os cerrados maranhenses recobrem a região oriental e meridional do Estado e constituem uma das regiões de cerrado menos conhecidas quanto à fauna, principalmente de invertebrados (REBÊLO \& CABRAL, 1997; Albuquerque \& Mendonça, 1999; RêGo et al., 2006). A maioria dos estudos sobre a fauna de abelhas do Maranhão abrange principalmente o norte do Estado (REBÊLO, 1995; GonÇALVES et al., 1996; BRITO \& RÊGO, 2001; Silva \& Rebêlo, 2002). A subtribo Meliponina (Hymenoptera, Apidae, Apini) possui aproximadamente 54 espécies inventariadas em diferentes ecossistemas no Estado (AlbuQuerque \& RÊGo, 2000). Apesar disso, há poucos trabalhos sobre espécies vegetais utilizadas para nidificação, bem como sobre a abundância destas em áreas de cerrado (ou mesmo em regiões semi-áridas do Brasil) (RêGo \& BRito, 1996; ANTONINI \& MARTins, 2003; MARTINS et al., 2004).

As abelhas sem ferrão são insetos eussociais e freqüentemente nidificam em ocos de árvores, ninhos de térmitas ou formigas, em cavidades subterrâneas ou expostos em galhos (CAMARGo, 1970; Wille \& Michener, 1973; Rоuвıк, 1983; Rouвıк, 1989). Os padrões de distribuição espacial e abundância de ninhos de insetos eussociais são bem estudados em formigas (WIERNASZ \& Cole, 1995), térmitas (Jones, 1993) e Apis spp. (McNally \& SCHNEIDER, 1996; OldRoyd et al., 1997). Em abelhas sem ferrão, porém, estes estudos são raros (MichENER, 1974; HubBell \& Johnson, 1977; HubBell \& Johnson, 1978). A compreensão do padrão de sua distribuição e abundância é importante para o desenvolvimento de estratégias de conservação que reduzam os efeitos da fragmentação de habitats sobre a estrutura genética e ecológica de populações de abelhas do grupo Meliponina (BREED et al., 1999).

O desaparecimento de polinizadores, resultado da degradação da fauna, pode impor prejuízos às populações de plantas (falha na reprodução, perda de diversidade genética ou decréscimo da progênie devido à depressão 
endogâmica) (JENNERSTEN, 1988; BAWA, 1990). Quaisquer alterações nos níveis de polinização podem afetar ainda diversos organismos que dependem direta ou indiretamente da polinização: animais que se utilizam de sementes e frutos produzidos pelas plantas, e animais que tem em sua dieta aqueles que dependem dos recursos vegetais (JANZEN, 1987). Assim, torna-se importante a conservação destas abelhas, já que são reconhecidas polinizadoras em ambientes naturais (HEARD, 1999).

Os invertebrados, particularmente os insetos, por responderem rapidamente a mudanças e distúrbios nos ecossistemas e contribuírem para sua sustentabilidade através de processos como decomposição, transferência de energia e polinização (RECHER et al., 1993; ANDERSEN \& SparLING, 1997), são freqüentemente usados como indicadores da qualidade do ambiente (PEARson \& Cassola, 1992; Hamer et al., 1997; Hill, 1999; Jones \& EgGleton, 2000; Kitching et al., 2000).

Muitos esquemas de conservação utilizam práticas de manejo da paisagem para conservar e realçar as comunidades florais, consequientemente melhorando a disponibilidade de alimento e recursos utilizados na construção dos ninhos de abelhas do grupo Meliponina. Entretanto, relativamente poucos esforços têm enfocado a necessidade de recursos para nidificação em áreas reduzidas (Роттs et al., 2005).

A redução de grandes extensões de cerrado em todo o Brasil (RATTER et al., 1997), torna imprescindível compreender aspectos da estrutura de comunidade de abelhas Meliponina para subsidiar a formulação de estratégias de conservação. Desta forma, o trabalho objetivou identificar as espécies de abelhas sem ferrão presentes em três áreas de cerrado no Maranhão. Também foi objetivo do trabalho identificar e caracterizar os substratos vegetais utilizados como locais de nidificação. Através destes propósitos, pretendeu-se averiguar a abundância e distribuição espacial de ninhos, a riqueza de espécies de abelhas e os padrões de uso dos substratos para nidificação.

\section{MATERIAL E MÉTODOS}

O trabalho foi realizado entre julho de 2001 e julho de 2004 em áreas de reserva pertencente à Comercial e Agrícola Paineiras Ltda, localizada no município de

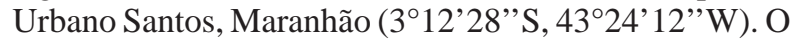
clima da região é tropical quente e úmido, com precipitação média anual de $1.500 \mathrm{~mm}$, estação chuvosa de janeiro a junho e de estiagem de julho a dezembro (Eiten, 1994).

As observações e coletas foram feitas mensalmente em três áreas de cerrado sensu stricto denominadas área 1 (41,9 ha), área 2 (29,6 ha) e área 3 (114 ha), que futuramente constituirão ilhas de vegetação natural interligadas por corredores ecológicos após implantação de projeto de silvicultura em porções vizinhas às áreas de estudo (Fig. 1). Foram feitas observações minuciosas em toda extensão das três áreas, por meio de deslocamentos ao longo de transectos paralelos para detectar os locais de nidificação de abelhas Meliponina (ninhos aéreos e ninhos no solo). Ao final de todas as coletas e observações toda a extensão das áreas havia sido vistoriada. Os ninhos encontrados foram numerados

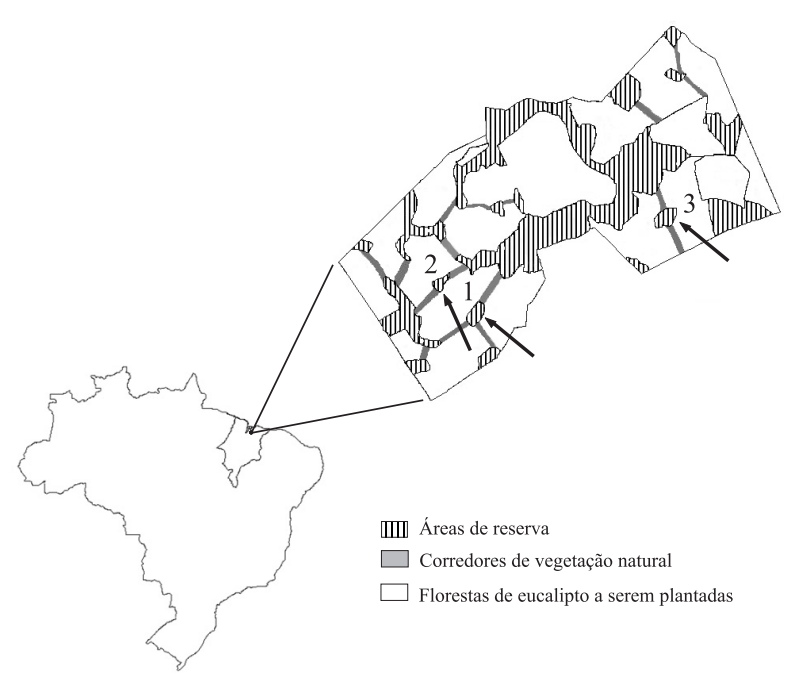

Fig. 1. Localização das áreas de estudo no município de Urbano Santos, nordeste do estado do Maranhão. As setas apontam para as áreas de coleta.

e georeferenciados. Coletaram-se amostras dos indivíduos de cada ninho e das espécies vegetais nas quais estes nidificaram para posterior identificação. As abelhas coletadas foram depositadas na coleção entomológica e as amostras das espécies vegetais no herbário da Universidade Federal do Maranhão (UFMA). Para caracterizar os substratos nidificados pelas abelhas mediu-se o diâmetro da árvore na altura do peito (DAP) e a altura da entrada do ninho em relação ao solo, utilizandose fita métrica.

Estimou-se o intervalo de confiança de $95 \%$ para o DAP das árvores ocupadas. Foi realizada análise de variância e teste de Tukey para amostras de tamanhos diferentes entre as médias de DAP das espécies vegetais que abrigavam ninhos. As espécies vegetais representadas por apenas um ou dois indivíduos e as espécies de abelhas representadas por um ou dois ninhos foram agrupadas para a análise. Utilizou-se o teste de Kruskal-Wallis para averiguar diferenças nas alturas das entradas dos ninhos entre as espécies de abelhas, bem como o teste de comparação múltipla. Verificou-se ainda, a distribuição espacial dos ninhos, através do índice de dispersão do vizinho mais próximo (R) (CLARK \& EVANS, 1954). As análises estatísticas foram realizadas através do programa R (R DeVelopMent CoRE TEAm, 2007), exceto os cálculos relacionados com o método do vizinho mais próximo que foram executados em planilha do Microsoft ${ }^{\circledR}$ Office Excel 2003.

\section{RESULTADOS}

Setenta e três ninhos de abelhas pertencentes a 15 espécies foram localizados nas três áreas estudadas. Dentre estes, ninhos de Partamona chapadicola Pedro \& Camargo, 2003 foram mais abundantes $(34,25 \%)$, seguidos por Oxytrigona sp. 2 (20,55\%) (Fig. 2, Tab. I). A espécie mais generalista com relação aos substratos utilizados (sete espécies vegetais) foi Oxytrigona sp. 2.

Foram identificadas 11 espécies vegetais utilizadas para construção dos ninhos: Qualea parviflora 
(Vochysiaceae), Salvertia convallariodora (Vochysiaceae), Caryocar brasiliense (Caryocaraceae), Tabebuia sp. (Bignoniaceae), Vatairea sp. (Fabaceae), Parkia platycephala (Fabaceae), Buchenavia sp. (Combretaceae), Jacaranda sp. (Bignoniaceae), Platonia insignis (Clusiaceae), Terminalia fagifolia (Combretaceae) e Salacia amygdalina (Hippocrateaceae). A freqüência de ninhos por tipo de substrato variou entre as espécies de abelhas. O substrato de nidificação mais frequiente foi $Q$. parviflora $(\mathrm{n}=28)$ com $38,36 \%$ do total de ninhos registrados. Esse substrato abrigou $72 \%$ dos ninhos de $P$. chapadicola $(n=25)$. O substrato no qual ocorreu maior diversidade de espécies, e onde se encontrou a maioria das espécies de abelhas com apenas um ninho registrado foi $S$. convallariodora.

A maioria dos ninhos de abelhas sem ferrão ocorreu em cavidades de árvores. Apenas Partamona spp. ocorreram associadas a ninhos de outros insetos. Ninhos de Partamona seridoensis Pedro \& Camargo, 2003 foram encontrados em termiteiros arborícolas externos, ativos, construídos de terra e localizados na base das árvores. Partamona chapadicola ocorreu em termiteiros, em ocos de troncos e em galhos de árvores vivas. Em três ocasiões

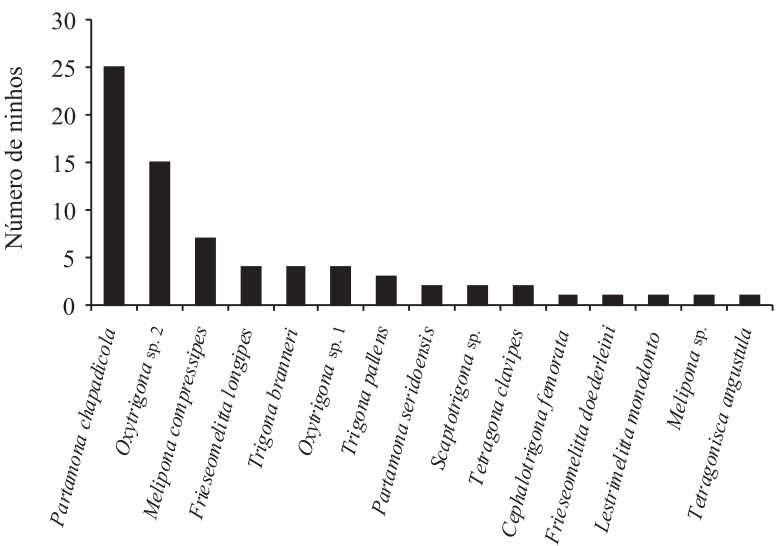

Fig. 2. Frequiência de espécies de abelhas Meliponina em três áreas de cerrado no município de Urbano Santos, Maranhão.

Tabela I. Abundância de espécies de abelhas Meliponina em três áreas de cerrado no município de Urbano Santos, Maranhão.

\begin{tabular}{lccc}
\hline Espécies de abelhas & \multicolumn{3}{c}{ Número de ninhos } \\
& Área 1 & Área 2 & Área 3 \\
\hline Partamona chapadicola & 4 & 2 & 19 \\
Oxytrigona sp. 2 & 6 & 4 & 5 \\
Melipona compressipes & 0 & 3 & 4 \\
Frieseomelitta longipes & 0 & 0 & 4 \\
Trigona branneri & 2 & 1 & 1 \\
Oxytrigona sp. 1 & 1 & 2 & 1 \\
Trigona pallens & 1 & 0 & 2 \\
P. seridoensis & 1 & 1 & 0 \\
Scaptotrigona sp. & 0 & 0 & 2 \\
Tetragona clavipes & 0 & 1 & 1 \\
Cephalotrigona femorata & 0 & 0 & 1 \\
Frieseomelitta doederleini & 1 & 0 & 0 \\
Lestrimelitta monodonta & 1 & 0 & 0 \\
Melipona sp. & 1 & 0 & 0 \\
Tetragonisca angustula & 0 & 1 & 0 \\
Total & 18 & 15 & 40 \\
\hline
\end{tabular}

encontraram-se mais de um ninho desta espécie em uma mesma árvore: dois ninhos em Q. parviflora e Vatairea sp., e dois ninhos de $P$. chapadicola mais um de Trigona pallens (Fabricius, 1798) em Q. parviflora. A ocorrência de mais de um ninho na mesma árvore também foi constatada para Oxytrigona spp. (Oxytrigona sp. 1 e Oxytrigona sp. 2 em S. convallariodora) e Melipona compressipes (Fabricius, 1804) (dois ninhos em S. convallariodora). Ninhos aéreos de Trigona branneri Cockerell, 1912 foram construídos sobre ramos de árvores e apoiados em forquilhas, e algumas espécies nidificaram próximo ao solo, na base de árvores: Tetragonisca angustula (Latreille, 1811), Melipona sp. e Trigona fulviventris Guérin, 1837.

A análise de variância entre o DAP médio das espécies de árvores com ninho indicou diferenças entre os grupos $(\alpha<0,05)$. Através do teste de Tukey, observouse que a média igual a $25,75 \mathrm{~cm}$ de Tabebuia sp. é inferior as médias de $C$. brasiliense, $(\bar{x}=44,06 \mathrm{~cm}), S$. convallariodora $(\bar{x}=43,22 \mathrm{~cm})$ e $P$. platycephala $(\bar{x}=50,32 \mathrm{~cm})$. O DAP médio de Tabebuia sp. mostrou-se marginalmente inferior $(\alpha=0,0544)$ ao de $Q$. parviflora $(\bar{x}=40,42 \mathrm{~cm})$ e semelhante a Vatairea $\mathrm{sp} .(\bar{x}=44,07 \mathrm{~cm})$. Segundo o teste de Kruskal-Wallis, as espécies de abelhas apresentaram diferenças em relação à altura da entrada dos ninhos $(\alpha<0,05)$. O teste de comparação múltipla indicou que T. branneri possui entrada mais alta $(\bar{x}=11,6 \mathrm{~m})$ do que $P$. chapadicola $(\bar{x}=2,82 \mathrm{~m})$, do que o grupo de espécies agregadas $(\bar{x}=1,44 \mathrm{~m})$ e do que T. pallens $(\bar{x}=0,59 \mathrm{~m})$. Já Oxytrigona sp. $1(\bar{x}=6,17 \mathrm{~m})$ tem entrada mais alta que T. pallens.

O padrão de dispersão dos ninhos de abelhas nas três áreas foi o seguinte: na área 1 os substratos nidificados distribuíram-se de forma aleatória $(\mathrm{R}=0,81 ; \mathrm{z}=-1,57$; p>0,05;n=18) (Fig. 3); na área 2 o padrão de dispersão também foi aleatório $(\mathrm{R}=0,89 ; \mathrm{z}=-0,78 ; \mathrm{p}>0,05 ; \mathrm{n}=15)$ (Fig. 4), e na área 3, os ninhos ocorreram uniformemente espaçados, com índice de dispersão do vizinho mais próximo igual a 1,88 (z=10,35; $\mathrm{p}<0,05 ; \mathrm{n}=40)$ (Fig. 5).
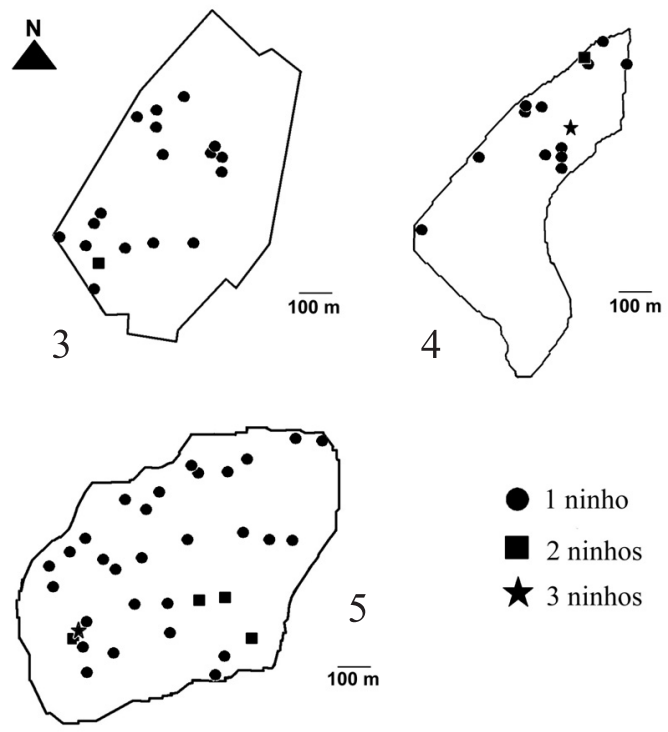

Figs. 3-5. Distribuição espacial dos ninhos de abelhas Meliponina em áreas de cerrado no município de Urbano Santos, Maranhão: 3 , aleatória, área $1 ; 4$, aleatória, área 2; 5, uniforme, área 3. 


\section{DISCUSSÃO}

A fauna de abelhas Meliponina encontrada inclui espécies cuja distribuição não havia sido relatada para o Maranhão. O gênero Frieseomelitta, até então representado por quatro espécies no Estado: $F$. flavicornis (Fabricius, 1798), F. varia (Lepeletier, 1836), F. portoi (Friese, 1900) e F. silvestrii (Friese, 1902) (REBÊLO et al., 2003) foi acrescido de mais duas espécies: $F$. longipes (Smith, 1854) e F. doederleini (Friese, 1900).

As espécies de abelhas encontradas nidificaram em diferentes espécies arbóreas; entretanto, $61,65 \%$ dos ninhos foram observados em apenas duas espécies de árvores: $Q$. parviflora e $S$. convallariodora. Martins et al. (2004) observaram utilização de 12 espécies arbóreas por meliponíneos na caatinga e $75 \%$ destes ninhos ocorreram em apenas duas espécies de árvores. RÊGO \& BRITO (1996) verificaram a utilização de apenas duas espécies vegetais para nidificação por meliponíneos em cerrado maranhense. Segundo aqueles autores, $S$. convallariodora foi a espécie vegetal que abrigou maior diversidade de espécies de abelhas sem ferrão e também o substrato de nidificação utilizado pela maioria dos meliponíneos (88\%; $\mathrm{n}=22$ ), sendo os demais ninhos encontrados em ocos de $P$. insignis.

Quando se compara as freqüências relativas dos substratos vegetais com ninhos, aqui observadas, com as frequiências destas espécies em outra área de cerrado no Maranhão (Imaña-Encinas \& De Paula, 2003), observam-se algumas diferenças. Salvertia convallariodora, segundo Imaña-Encinas \& De Paula (2003), ocorre com frequiência relativa de $11,42 \%$. No entanto, foi verificado seu uso como local de nidificação, em 25,71\% das observações. Por outro lado, Parkia platycephala e Platonia insignis, ocorreriam no cerrado maranhense com freqüências de 15,87\% e 7,3\% respectivamente (ImaÑa-Encinas \& De PaUla, 2003), mas, foram observadas contendo ninhos em $4,28 \%$ e $1,42 \%$ das observações. As diferenças entre freqüência de utilização das espécies vegetais pelas abelhas, e a frequiência de ocorrência destas árvores no cerrado maranhense podem ser explicadas pela longevidade distinta das espécies arbóreas. As espécies de abelhas encontradas tenderiam a nidificar em árvores com DAP médio entre $36,21 \mathrm{~cm}$ e $41,68 \mathrm{~cm}$, representando árvores mais velhas (Aquino et al., 2007). Desta forma, $S$. convallariodora poderia ter maior longevidade, permitindo maior crescimento, ao contrário de $P$. insignis que possui longevidade menor. Logo, idades maiores implicam em maiores chances de formação de cavidades. Em acréscimo, Imaña-Encinas \& De Paula (2003) verificaram uma frequiência relativa de $39,04 \%$ para $Q$. parviflora, sendo a frequiência de árvores com ninho de $34,28 \%$. Estas frequiências podem ser consideradas similares e ao relacioná-las ao fato de que $Q$. parviflora possui baixa longevidade, porém sua taxa de recrutamento de novos indivíduos para geração seguinte tende a ser elevada, o que torna a densidade desta árvore significativa nas áreas de cerrado em que ocorre (IMAÑA-
Encinas \& De Paula, 2003; Aquino et al., 2007). Tabebuia sp., Q. parviflora e S. convallariodora contiveram 62,89\% dos ninhos, e, segundo Aquino et al. (2007), possuem extensa longevidade. A análise de variância indicou que Tabebuia sp. tende a possuir o menor DAP médio. Contudo, estes valores são maiores que a média de $11,35 \mathrm{~cm}$ apresentada por Imaña-Encinas \& De Paula (2003). Mesmo com a tendência de possuir o menor valor médio de DAP, a análise de variância aponta que este valor possui chance de estar entre os valores do intervalo de confiança. Se o padrão for o mesmo observado por Aquino et al. (2007), as árvores com DAP do intervalo de confiança têm uma freqüência baixa, indicando que os locais para nidificação por abelhas Meliponina tendem a ser um recurso escasso no cerrado do Maranhão. Ainda é possível propor que não apenas a longevidade contribui para uma maior freqüência de nidificação em dada espécie de árvore, mas também as taxas de crescimento. AQuino et al. (2007), apontam que $Q$. parviflora e $S$. convallariodora (que continham $62,82 \%$ dos ninhos) tiveram taxas de crescimento de maior magnitude. Outro fator relevante é que a nidificação em dada espécie de árvore dependerá também da natureza da fibra da madeira. Algumas espécies arbóreas possuem fibra mais resistente e, portanto, menos susceptíveis a formarem cavidades.

Em geral, a estrutura interna dos ninhos de meliponíneos em troncos de árvore fica próxima à entrada dos ninhos (WiLle \& Michener,1973), assim a altura da entrada é uma referência para a altura dos ninhos como um todo. O teste de comparação múltipla sugere a construção preferencial do ninho em posições mais baixas por T. pallens e em posições mais altas por T. branneri. Porém, a maioria dos pares comparados não se diferenciou, indicando que a altura dos ninhos nas árvores possa ser dada ao acaso.

O padrão de distribuição espacial dos ninhos em abelhas sociais é pouco conhecido. Possivelmente, está relacionado a fatores extrínsecos como à distribuição e densidade de substratos adequados à construção destes, aos aspectos ecológicos de ocupação dos ocos e à predação, bem como aos aspectos intrínsecos de cada espécie (como a enxameação próximo à colônia mãe). O padrão de dispersão uniforme dos ninhos observados na área 3 pode ser devido a esta área ser mais impactada (maior ocorrência de incêndios durante o período de estudo e maior interferência antrópica), possuir mais trechos de vegetação de capoeira (quando comparada às áreas 1 e 2) e, possivelmente, possuir menor disponibilidade de recursos (florais e de nidificação). Já o padrão aleatório dos ninhos observados nas áreas $1 \mathrm{e}$ 2 , pode estar relacionado ao fato destas áreas possuírem vegetação mais densa e possivelmente apresentarem maior disponibilidade de recursos. HubBell \& Johnson (1977), argumentam que competição agressiva por alimento seria a razão para dispersão uniforme em meliponíneos neotropicais que forrageiam em grupo e que o espaçamento entre ninhos seria mediado por encontros agressivos entre colônias que competem por um novo sítio de nidificação. Por conseguinte, o padrão 
aleatório encontrado nas áreas 1 e 2 pode indicar ausência (ou baixo nível) de competição por recursos. Uma vez que os recursos estejam disponíveis para as populações, a dispersão de novas colônias até novos sítios de nidificação ocorreria ao acaso. $\mathrm{Ou}$, alternativamente, a distribuição aleatória poderia indicar que as fontes de recursos também estariam dispersas aleatoriamente.

Os resultados gerados neste trabalho certamente auxiliarão os projetos de conservação e contribuirão para uma melhor compreensão da organização das comunidades do cerrado. Os recursos para nidificação, em adição aos recursos disponíveis para forrageamento e outras dinâmicas da estrutura de comunidades de abelhas Meliponina também necessitam ser considerados para uma melhor avaliação das consequiências da redução e isolamento de populações em fragmentos, permitindo o estabelecimento de programas efetivos de manejo em áreas reduzidas de floresta.

Agradecimentos. Ao PIBIC (Programa Institucional de Bolsas de Iniciação Científica) e CNPq (Conselho Nacional de Desenvolvimento Científico e Tecnológico) pelo auxílio financeiro. À Comercial e Agrícola Paineiras Ltda pelo apoio logístico. Ao professor Gabriel Melo (UFPR) pela identificação das espécies de abelhas. Ao professor Paulo Figueiredo (UFMA) pela identificação das espécies vegetais. Aos senhores Nonato e "Codó" pela assistência em campo.

\section{REFERÊNCIAS BIBLIOGRÁFICAS}

Albuquerque, P. \& MendonçA, J. A. 1999. Anthophoridae (Hymenoptera; Apidae) e flora associada em uma formação de cerrado no município de Barreirinhas, MA, Brasil. Acta Amazônica 26:45-54.

Andersen, A. N. \& Sparling, G. P. 1997. Ants as indicators of restoration success: relationship with soil microbial biomass in the Australian seasonal tropics. Restoration ecology 5(2): 109-114.

Antonini, Y. \& Martins, R. P. 2003. The value of a tree species (Caryocar brasiliense) for a stingless bee Melipona quadrifasciata quadrifasciata. Journal of Insect Conservation 7(3): 167-174.

Aquino, F. G.; Walter, B. M. T. \& Ribeiro, J. F. 2007. Woody community dynamics in two fragments of "cerrado" stricto sensu over a seven-year period (1995-2002), MA, Brazil. Revista Brasileira de Botânica 30(1):113-121.

BAWA, K. S. 1990. Plant-pollinator interactions in tropical rain forests. Annual Review of Ecology and Systematics 21:399-422.

Breed, M. D.; McGlynn, T. P.; Sanctuary, M. D.; Stocker, E. M. \& CRUZ, R. 1999. Distribution and abundance of colonies of selected meliponine species in a Costa Rican tropical wet forest. Journal of Tropical Ecology 15(6):765-777

Brito, C. M. DE \& RÊGo, M. M. C. 2001. Community of male Euglossini bees (Hymenoptera, Apidae) in a Secondary Forest, Alcântara - MA, Brazil. Brazilian Journal of Biology 61(4):631-638.

Camargo, J. M. F. 1970. Ninhos e biologia de algumas espécies de Meliponídeos (Hymenoptera: Apidae) da região de Porto Velho, Território de Rondônia, Brasil. Revista de Biologia Tropical 16(2):207-239.

Clark, P. \& Evans, F. 1954. Distance to nearest neighbor as a measure of spatial relationships in populations. Ecology 35(4): 445-453.

Eiten, G. 1994. Duas travessias na vegetação do Maranhão. Brasília, Sucesso. 72p.

Goncalves, S. J. M.; RêGo, M. \& AraúJo, A. 1996. Abelhas sociais (Hymenoptera: Apidae) e seus recursos florais em uma região de mata secundária, Alcântara, Maranhão, Brasil. Acta Amazônica 26:55-68.
Hamer, K. C.; Hill, J. K. Lace, L. A. \& Langan, A. M. 1997. Ecological and biogeographical effects of forest disturbance on tropical butterflies of Sumba, Indonesia. Journal of Biogeography 24(1):67-75.

Heard, T. A. 1999. The role of stingless bees in crop pollination. Annual Review of Entomology 44:183-206.

Hill, J. K. 1999. Butterfly spatial distribution and habitat requirements in a tropical forest: impacts of selective logging. Journal of Applied Ecology 36(4):564-572.

Hubbell, S. P. \& Johnson, L. K. 1977. Competition and nest spacing in a tropical stingless bee community. Ecology 58(5):949-963.

1978. Comparative foraging behavior of six stingless bee species exploiting a standardized resource. Ecology 59(6): 1123-1136.

Imaña-Encinas, J. \& De Paula, J. E. 2003. Análise da vegetação de cerrado no município de Santa Quitéria - Maranhão. Brasil Florestal 23(78):33-42.

JANZEN, D. H. 1987. Insect diversity of a Costa Rica dry forest: why keep it, and how? Biological Journal of the Linnean Society 30:343-356.

Jennersten, O. 1988. Pollination in Dianthus deltoides (Caryophylaceae): effects of habitat fragmentation on visitation and seed set. Conservation Biology 2:359-366.

Jones, D. T. \& EgGleton, P. 2000. Sampling termite assemblages in tropical forests: testing a rapid biodiversity assessment protocol. Journal of Applied Ecology 37(1):191-203.

JoNES, S. C. 1993. Field observations of intercolony aggression and territory changes in Heterotermes aureus (Isoptera: Rhinotermitidae). Journal of Insect Behavior 6(2):225-236.

Kitching, R. L.; OrR, A. G.; Thalib, L.; Mitchell, H.; Hopkins, M. S. \& Graham, A. W. 2000. Moth assemblages as indicators of environmental quality in remnants of upland Australian rain forest. Journal of Applied Ecology 37(2):284-297.

Martins, C. F.; Cortopassi-Laurino, M.; Koedam, D. \& ImperatrizFonsecA, V. L. 2004. Espécies arbóreas utilizadas para nidificação por abelhas sem ferrão na caatinga (Seridó, PB; João Câmara, RN). Biota Neotropica 4(2):1-8

McNally, L. C. \& Schneider, S. S. 1996. Spatial distribution and nesting biology of colonies of the African honey bee Apis mellifera scutellata (Hymenoptera: Apidae) in Botswana, Africa. Environmental Entomology 25:643-652.

Michener, C. D. 1974. The social behavior of the bees: a comparative study. Cambridge \& Massachusetts, Harvard University. 404p.

Oldroyd, B. P.; Thexton, E. G.; Lawler, S. H. \& Crozier, R. H. 1997. Population demography of Australian feral bees (Apis mellifera). Oecologia 111(3):381-387.

Pearson, D. L. \& Cassola, F. 1992. World-wide species richness patterns for tigerbeetles (Coleoptera: Cincidelidae): indicator taxon for biodiversity and conservation studies. Conservation Biology 6:376-391.

Potts, S. G.; Vulliamy, B.; Roberts, S.; O’Toole, C.; Dafni, A.; Ne'eman, G. \& Willmer, P. 2005. Role of nesting resources in organizing diverse bee communities in a Mediterranean landscape. Ecological Entomology 30:78-85.

Ratter, J. F.; Ribeiro, J. F. \& Bridgewater, S. 1997. The Brazilian cerrado vegetation and threats to its biodiversity. Annals of Botany 80:223-230

R Development Core Team. 2007. R: A language and environment for statistical computing. Disponível em: <http://www.R-project.org > Acesso em: 15.10.2007.

RebÊLo, J. M. M. 1995. Espécies de Anthophoridae (Hymenoptera, Apoidea) e sua associação com flores, numa área restrita da ilha de São Luís - MA, Brasil. Boletim do Museu Paraense Emílio Goeldi, série Zoologia 11(2):105-124.

Rebêlo, J. M. M. \& CAbral, A. J. 1997. Espécies de Euglossinae (Hymenoptera, Apidae) de Barreirinhas, Zona do Litoral da Baixada Oriental Maranhense. Acta Amazônica 27(2):145-152.

Rebêlo, J. M. M.; RêGo, M. M. C. \& Albuquerque, P. M. C. 2003. Abelhas (Hymenoptera, Apoidea) da região setentrional do Estado do Maranhão, Brasil. In: Melo, G. A. R \& Alves-dosSantos, I. eds. Apoidea Neotropica: Homenagem aos 90 Anos de Jesus Santiago Moure. Criciúma, UNESC. p.265-278. 
Recher, H. F.; Hutchings, P. A. \& Rose, S. 1993. The biota of the Hawkesbury-Nepean catchment: reconstruction and restoration. The Australian Zoologist 29(1-2):3-41.

Rêgo, M. M. C; Albuquerque, P. M. C; Ramos, M. C. \& Carreira, L. M. 2006. Aspectos da biologia de nidificação de Centris flavifrons (Friese) (Hymenoptera: Apidae, Centridini), um dos principais polinizadores do murici (Byrsonima crassifolia L. Kunth, Malpighiaceae), no Maranhão. Neotropical Entomology 35(5):579-587.

RÊGo, M. M. C. \& BRito, C. 1996. Abelhas sociais (Apidae: Meliponini) em um ecossistema de cerrado s.l. (Chapadinha MA, BR): distribuição dos ninhos. In: Encontro Sobre Abelhas, $3^{\circ}$, Ribeirão Preto, Anais..., Ribeirão Preto, FFCLRP-USP. v.3, p.238-247.
RoubiK, D. W. 1983. Nests and colony characteristics of stingless bees from Panama. Journal of the Kansas Entomological Society 56(3):327-355.

1989. Ecology and Natural History of Tropical Bees. Cambridge, Cambridge University. 514p.

Silva, F. S. \& Rebêlo, J. M. M. 2002. Espécies de Euglossinae (Hymenoptera, Apidae) da ilha do Cajual, zona do Golfão maranhense. Brazilian Journal of Biology 29(4):587-599.

Wiernasz, D. C \& Cole, B. J. 1995. Spatial distribution of Pogonomyrmex occidentalis: recruitment, mortality and overdispersion. Journal of Animal Ecology 64(4):519-527.

Wille, A. \& Michener, C. D. 1973. The nest architecture of stingless bees with special reference to those of Costa Rica (Hymenoptera: Apidae). Revista de Biologia Tropical 21(1):9-278

Recebido em dezembro de 2007. Aceito em julho de 2008. ISSN 0073-4721

Artigo disponível em: www.scielo.br/isz 